\title{
Correspondence
}

\section{Re: Role of robot-assisted laparoscopy in adjuvant surgery for locally advanced cervical cancer (EJSO 2010; 36(4):409-413) by Lambaudie E. et al.}

\section{Dear Editor,}

We read with interest the recent article by Lambaudie E. et al. ${ }^{1}$ who compared 22 cases of robot-assisted laparoscopy with 20 cases of laparotomy and 16 cases of conventional laparoscopy in patients with locally advanced cervical cancer. Treatment included aortic lymph node staging before chemoradiation (CT/RT) to define the radiation field, brachytherapy, followed by robot-assisted surgery (simple hysterectomy with lymphadenectomy).

In the paper the authors affirm that radical hysterectomy was performed systematically only in the conventional laparoscopy group or if difficulties were encountered during the surgical procedure in exposing surgical landmarks (ureter, uterine pedicle) due to fibrosis and retraction after concomitant chemoradiation and brachytherapy. How many simple hysterectomies have been converted into radical hysterectomy during surgery? Which kind of radical hysterectomy do they perform? Why the robot-assisted laparoscopy and traditional laparotomy groups underwent different surgical treatments?

Recent observations suggested that radical surgery tends to be associated with a lower rate of local recurrence than extrafascial hysterectomy and no surgery at all and that completion surgery carries out a better prognosis for patients with bulky residual tumor after CT/RT. ${ }^{2}$

We think that the robot-assisted surgery can provide an advantage in performing difficult steps of radical surgery above all in dissecting and unroofing of the ureter. We address the potential benefits of the robotic system in reducing surgeon fatigue and simplifying the difficult procedural steps of radical hysterectomy.

In the comparison of surgical procedures, authors observed a significance difference in terms of per-operative criteria between the two groups. This difference was explained by the surgical approach used. What is the relationship between the preoperative staging and the modulation

DOI of original article: 10.1016/j.ejso.2009.12.004. of radicality? Also because we observed that the median number of lymph nodes removed was low. We believe that the robotic system can aid in performing a meticulous dissection for extended pelvic and para-aortic lymphadenectomy during the surgical procedure for gynecologic cancer as also reported in a recent case-matched analysis of robot-assisted radical hysterectomy compared with laparoscopy and laparotomy was published by Estape et al. ${ }^{3}$ The highest number of resected lymph nodes was shown in the robotic group (32.4 for robotic surgery, 18.6 for laparoscopy, and 25.7 for laparotomy), and the authors estimated a decrease in the rate of intraoperative injuries from $11.8 \%$ to $3.1 \%$ with the introduction of robotics.

In the follow-up section, the authors affirm that there was no difference in recurrence rate between robotassisted laparoscopy, conventional laparoscopy and laparotomy. Where patients have relapsed? Colombo et al., ${ }^{4}$ in the first experience of total laparoscopic radical hysterectomy for locally advanced cervical carcinoma after concurrent chemoradiation therapy, showed $8.7 \%$ of microscopic or macroscopic residual disease due to incomplete resection. Have you had close or positive surgical margins in the different groups? What kind of treatment you using in these cases?

As concerned Table $3^{1}$ content we noted that in the three arms the authors obtained a low rate of persistence of cervical residual tumour, above all in conventional laparoscopy group. The rate of persistence of residual tumour after CT/RT is undoubtedly not negligible, varying between $35 \%$ and $63 \%$. Which kind of protocol do they use for neoadjuvant RT/CT treatment?

In conclusion, we believe that the use of the robot in surgical procedures continues to increase. However, reports concerning the long-term survival in patients who have undergone robot-assisted radical hysterectomy are yet to be discovered and it is crucial that more studies be conducted to further confirm the feasibility and safety of this technology in different types of procedures.

\section{Conflict of interest}

We declare there are no financial or other relationships that may lead to a conflict of interest. 


\section{References}

1. Lambaudie E, Narducci F, Bannier M, et al. Houvenaeghel G.Role of robot-assisted laparoscopy in adjuvant surgery for locally advanced cervical cancer. Eur J Surg Oncol 2010;36(4): 409-13.

2. Motton S, Houvenaeghel G, Delannes M, et al. Results of surgery after concurrent chemoradiotherapy in advanced cervical cancer: comparison of extended hysterectomy and extrafascial hysterectomy. Int J Gynecol Cancer 2010;20:268-75.

3. Estape R, Lambrou N, Diaz R, et al. A case matched analysis of robotic radical hysterectomy with lymphadenectomy compared with laparoscopy and laparotomy. Gynecol Oncol 2009;113:35761.

4. Colombo PE, Bertrand MM, Gutowski M, et al. Total laparoscopic radical hysterectomy for locally advanced cervical carcinoma (stages IIB, IIA and bulky stages IB) after concurrent chemoradiation therapy: surgical morbidity and oncological results. Gynecol Oncol 2009;114(3): 404-9.
E. Vizza*

Gynecologic Oncology Unit,

National Cancer Institute "Regina Elena", Via E. Chianesi 53, 00144 Rome, Italy

L. Patrizi

M. Saltari

Department of Surgery, University of Rome Tor Vergata, Rome, Italy

G. Corrado

Gynecologic Oncology Unit, Catholic University, Campobasso, Italy

E-mail address: giacomo.corrado@alice.it

*Corresponding author. Tel.: +3906 52666970;

fax: +3906 52666974

E-mail address: e.vizza@ifo.it

Available online 6 December 2011 